Portland State University

PDXScholar

10-2016

\title{
Hitting the Goalpost: Calculating the Fine Line Between Winning and Losing a Penalty Shootout
}

Ralf Widenhorn

Portland State University

Follow this and additional works at: https://pdxscholar.library.pdx.edu/phy_fac

Part of the Physics Commons

Let us know how access to this document benefits you.

Citation Details

Widenhorn, R. (2016). Hitting the Goalpost: Calculating the Fine Line Between Winning and Losing a Penalty Shootout. Physics Teacher, 54(7), 434-438.

This Article is brought to you for free and open access. It has been accepted for inclusion in Physics Faculty Publications and Presentations by an authorized administrator of PDXScholar. Please contact us if we can make this document more accessible: pdxscholar@pdx.edu. 


\section{Hitting the Goalpost: Calculating the Fine Line Between Winning and Losing a Penalty Shootout}

Ralf Widenhorm, Portland State University, Portland, OR

\section{he Portland Timbers \\ won their}

first Major League Soccer (MLS) Cup Championship in December 2015. However, if it had not been for a kind double goalpost miss during a penalty shootout a few weeks earlier, the Timbers would never have been in the finals. On Oct. 30th, after what has been called "the greatest penalty kick shootout in MLS history," featuring a combined 22 penalties that included penalties by both goalkeepers, the Timbers won their first-round playoff against Sporting Kansas City. ${ }^{1-2}$ During the thrilling shootout, which can be watched, for example, for example on the MLS website, Sporting had two potentially game-winning penalties miss by the smallest of margins. ${ }^{3}$ One penalty bounced off the goalpost back into the field and another was an improbable double post miss. For a physicist, this prompts an interesting research question. Could we find an estimate by what distance the double post penalty shown in Fig. 1 failed to be the game winning shot?

\section{Analysis}

In this manuscript we will use a geometrical analysis to develop the equations that describe the conditions for the ball ricocheting off both goalposts. We know intuitively that the penalty missed by a very small margin, but it is not trivial to get a good estimate by how much it was off. Even the smallest change could have made the difference between winning and losing. For example, the video shows that the ball bounces once as it travels from the left to the right goalpost. Unevenness in the ground could have nudged the ball ever so slightly

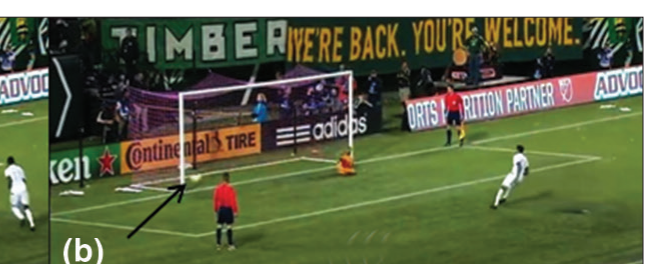

(b)
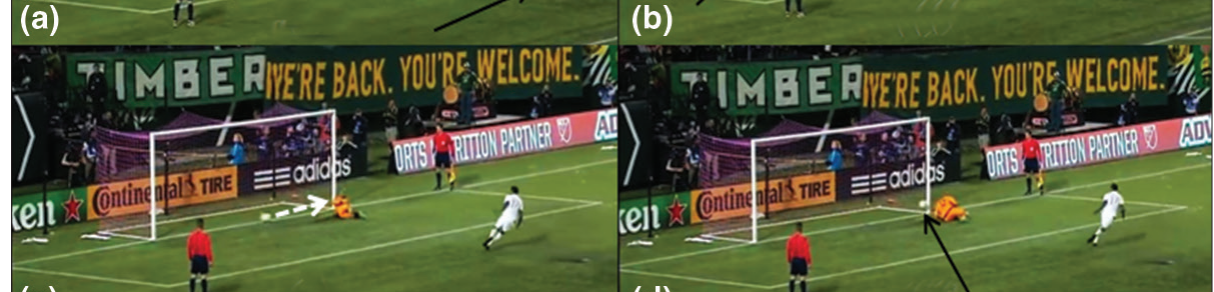

(d)

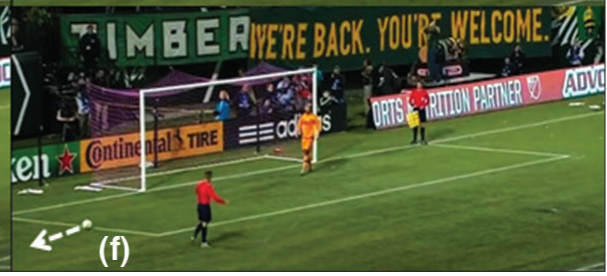

Fig. 1. Sequence of the double post penalty with black arrows indicating the location of the ball and white dashed arrows indicating the direction the ball ricocheted. (a) Player is getting ready to shoot the penalty. (b) Ball just before it bounces off the left post. The goal traveling toward the right post. (d) Ball as it bounces off the right post. The goalkeeper did not have enough time to recover and get to the ball. (e) Ball after it bounces off the right post. (f) Ball crosses the left goal box line. (Courtesy, Major League Soccer) from its straight path. A solvable model would require perfect knowledge of the surface structure or assume it to be flat. Like every messy real world problem, we need to start with a model based on some simplified assumptions and the need to discuss the validity of this model. For our model, we ignore the spin of the ball and potential unevenness of the Astroturf surface, and assume the ball does not curve and keeps its spherical shape as it bounces off a single contact point on the post. This simplified model reduces the motion of the ball to an analysis of reflection angles not unlike the analysis used to trace light rays reflecting off curved mirrors using basic trigonometry.

One needs to be conscious of the differences of this model to the actual physical system and know its limitations. Spin will cause a ball to curve due to the Magnus force and will impact the bounce upon hitting the goalpost or ground. Even if the penalty is shot without initial spin, friction will cause the ball to acquire a counterclockwise spin as viewed from the top upon bouncing off the left goalpost. ${ }^{4-8}$ The effect of spin on the trajectory of balls in sports ${ }^{9-11}$ has been studied widely, but would result in a much more complex model. Qualitatively we know that the Magnus force due to the counterclockwise spin would cause the ball to curve toward the goal. While it is difficult to observe a curving or unusual bounce of the ball in the video of this penalty, there are examples in soccer when it had a major impact on the outcome. For example, the effect of spin tends to be especially pronounced for a ball that bounces off the ground after being deflected hard off the 


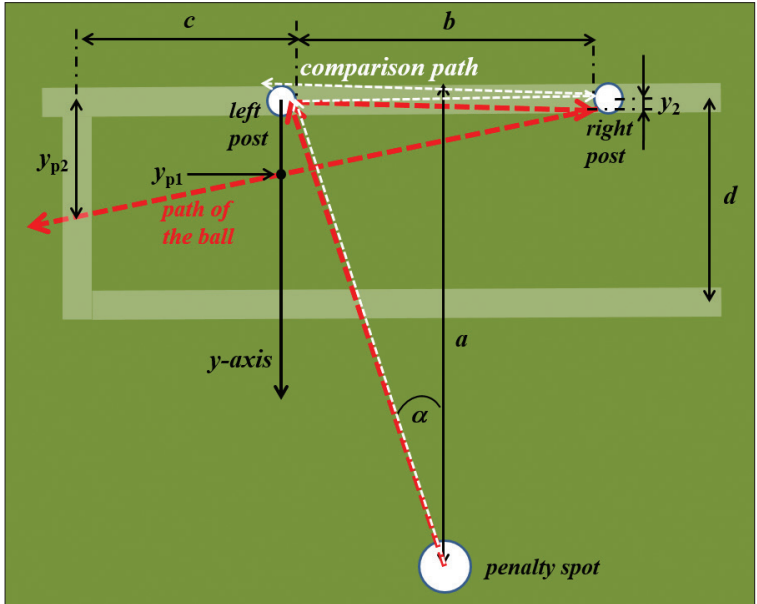

Fig. 2. Schematic of the ball path and relevant dimensions. The red dashed lines show the actual double bounce penalty and the white dashed lines show a hypothetical double bounce penalty that would have resulted in a goal.

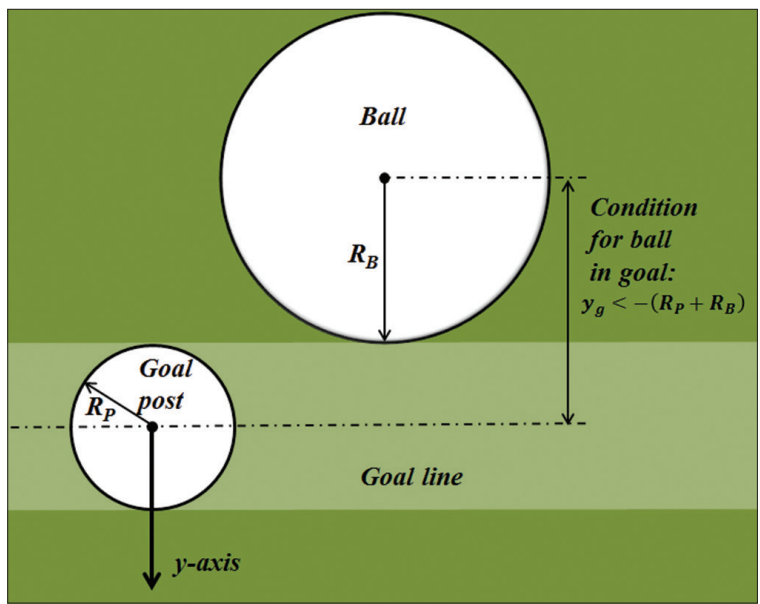

Fig. 3. Condition for a goal: The entire ball must cross the entire goal line.

crossbar and this led to questionable decisions by the referees in several World Cup games. ${ }^{12,13}$ Even though the spin is going to shift reflection angles toward a goal, the comparison of angles and distances for different scenarios is much more robust and can provide good order of magnitude estimates if we assume that parameters like spin and ball compression do not vary. Therefore, while the graphs contain absolute values for illustrative purposes, the reader should be aware that spin would shift those numerical values. In this study we will interpret only the difference between values and assume spin and all parameters except for the location of the ball remain constant. We will assume that the dimensions of the goal, ball, and soccer field shown in Fig. 2 are as stated by the Fédération Internationale de Football Association (FIFA) guidelines. ${ }^{14}$

- The distance between insides of the goalposts is: $b=7.32 \mathrm{~m}(8 \mathrm{yd})$.

- The distance from the penalty spot to the goal is: $a=11 \mathrm{~m}(12 \mathrm{yd})$.

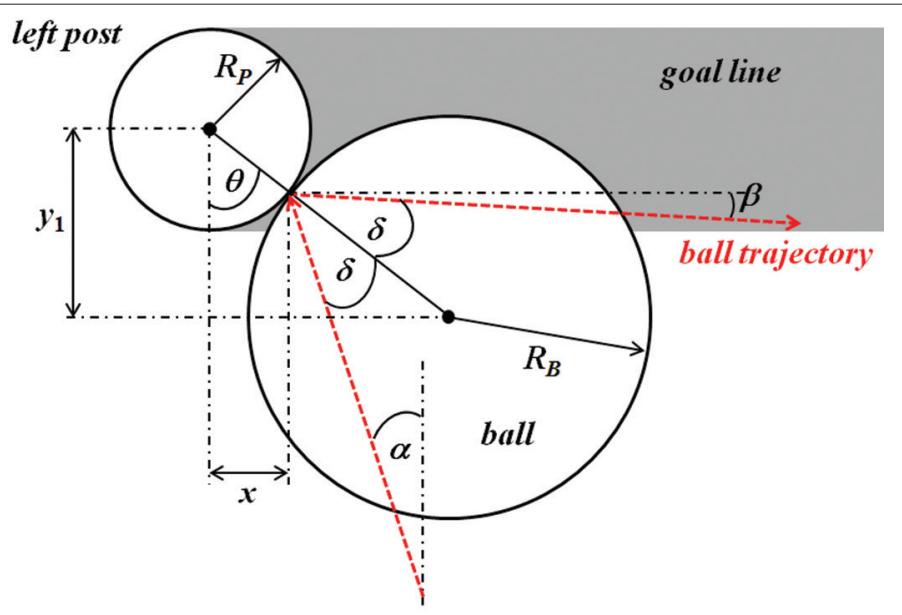

Fig. 4. Schematic of the angles and parameters as the ball hits the inside of the left goalpost.

- The dimensions of the goal area are: $c=d=5.5 \mathrm{~m}(6 \mathrm{yd})$.

- The goal lines must be of the same width as the goalposts, which do not exceed 120-mm (5-in) diameter. We will assume a common round $100-\mathrm{mm}$ (4-in) diameter aluminum post and a ball that satisfies the FIFA regulation of a circumference of $680-700 \mathrm{~mm}: R_{\mathrm{P}}=50 \mathrm{~mm}$ and $R_{\mathrm{B}}=$ $110 \mathrm{~mm}$.

Setting the direction of the positive $y$-axis toward the opponent's goal and its origin at the center of the left or right goalposts, we get that the condition for a goal (see Fig. 3) is

$$
y_{g}<-\left(R_{\mathrm{P}}+R_{\mathrm{B}}\right) . \quad \text { General condition for a goal }
$$

\section{- Ricochet off the left post}

The angle $\alpha$ of the incoming ball ignores the uncertainty of the exact location of the ball on the penalty spot and the slight distance from the inside of the post, and is calculated as

$$
\tan \alpha \approx \frac{b / 2}{\alpha} .
$$

Figure 4 shows the relevant distances and angles as the ball hits the left post. The angles of incidence and reflection $\delta$ are measured with respect to the normal to the goalpost and are assumed to follow the law of reflection in the same form as a light ray reflecting off a convex mirror. To find the normal to the post surface where the ball hit the post, we calculate

$$
\sin \theta=\frac{x}{R_{\mathrm{P}}} .
$$

The incident and reflected angles $\delta$ are related to $\theta$ and $\alpha$ as

$$
\delta=\theta-\alpha
$$

To find the location of the ball as it travels toward the right post, we calculate the angle $\beta$, which is measured with respect to the goal line as

$$
\beta=90^{\circ}-(2 \delta+\alpha) .
$$




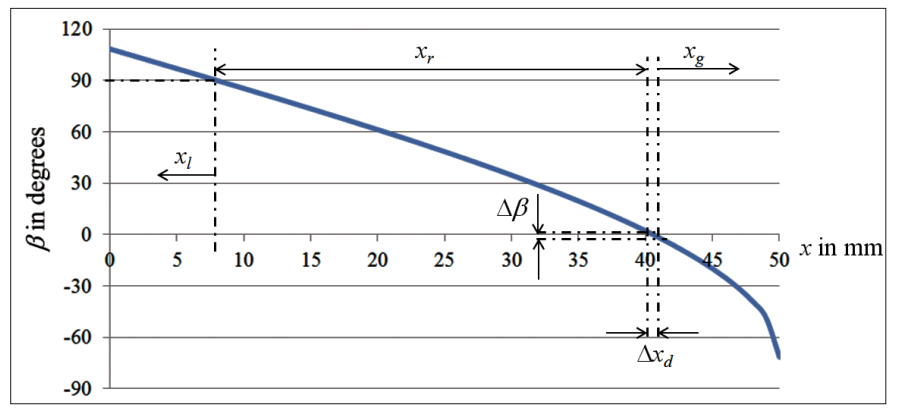

Fig. 5. Reflection angle $\beta$ as a function of $x$ after the ball bounces off the left post. $x_{1}$ and $x_{\mathrm{r}}$ are the $x$-values for which the ball bounces to the left $\left(x_{1}\right)$ and right $\left(x_{r}\right)$ part of the soccer field after hitting the post. For $x_{\mathrm{g}}$ the ball bounces into the goal without hitting the right post and the ball hits the right post for $x$-values within $\Delta x_{d}$.

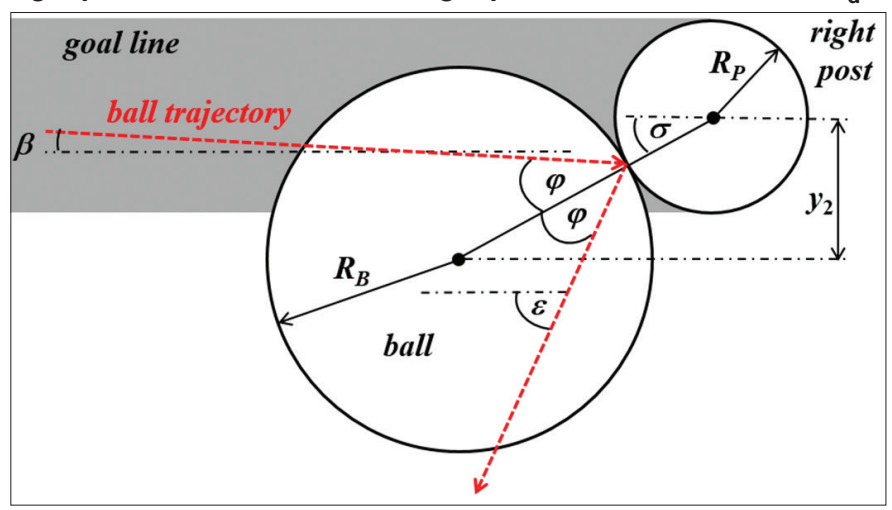

Fig. 6. Schematic of the angles and parameters as the ball hits the inside of the right goalpost.

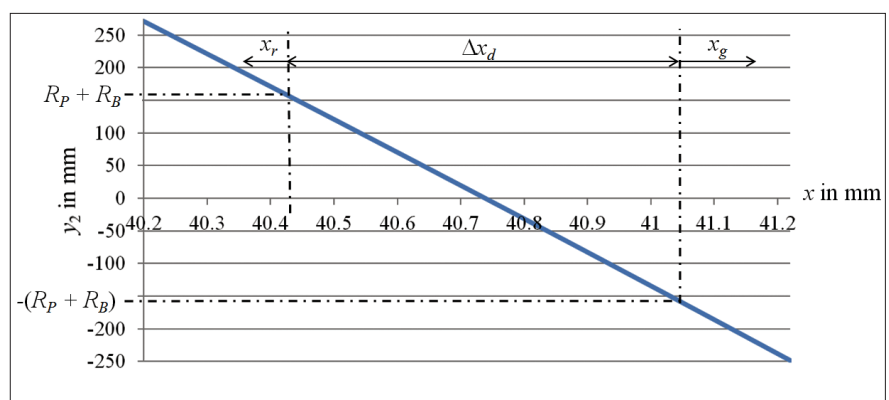

Fig. 7. $y_{2}$ as a function of $x$ as the ball reaches the right post. $x_{\mathrm{r}}$ are the $x$-values for which the ball bounces back into the field after hitting the post. For $x_{\mathrm{g}}$ the ball bounces into the goal without hitting the right post and the ball double bounces for $x$-values within $\Delta x_{d}$.

Inserting Eqs. (2), (3), and (4) into Eq. (5), we get $\beta$ as a function of the distance from the contact point to the center of the post $x$ as

$$
\beta=90^{\circ}-2 \theta+\alpha=90^{\circ}-2 \sin ^{-1}\left(\frac{x}{R_{\mathrm{p}}}\right)+\tan ^{-1}\left(\frac{b / 2}{\alpha}\right) .
$$

Note that since $a, b \gg x$, we ignore the slight $x$ dependence of $\alpha$ in Eq. (2), resulting in $\beta$ in Eq. (6) depending only on the $x$ in the inverse sine function.

Figure 5 shows $\beta$ as a function of $x$ as calculated by Eq. (6) from a bounce at the front midpoint of the post $(x=0 \mathrm{~mm})$ to the right inside edge $(x=50 \mathrm{~mm})$. For $\beta>90^{\circ}$ the ball bounces away from the shooter to the left side of the soccer field.
For other values of $x$, the ball moves toward the right side of the soccer field, following the goal line at $\beta=0^{\circ}$. For all negative values of $\beta$ the ball moves toward the back of the net.

From Fig. 4, Eq. (3), and a trigonometric identity, we find that the $y$-location of the ball on the left post can be calculated as

$$
\begin{aligned}
y_{1} & =\left(R_{\mathrm{P}}+R_{\mathrm{B}}\right) \cos \theta=\left(R_{\mathrm{P}}+R_{\mathrm{B}}\right) \cos \left[\sin ^{-1}\left(\frac{x}{R_{\mathrm{P}}}\right)\right] \\
& =\left(R_{\mathrm{P}}+R_{\mathrm{B}}\right) \sqrt{1-\left(\frac{x}{R_{\mathrm{P}}}\right)^{2}} .
\end{aligned}
$$

\section{- Double post bounce?}

Next, we will analyze the motion of the ball after it bounces off the left post and moves toward the right post. The ball travels the length of the goal, reaching the right post at $y_{2}$ from its center (see Fig. 6). Using Eqs. (6) and (7), we get

$$
\begin{aligned}
& y_{2}=y_{1}+b \tan \beta=\left(R_{\mathrm{P}}+R_{\mathrm{B}}\right) \sqrt{1-\left(\frac{x}{R_{\mathrm{P}}}\right)^{2}} \\
& +b \tan \left[90^{\circ}-2 \sin ^{-1}\left(\frac{x}{R_{\mathrm{P}}}\right)+\tan ^{-1}\left(\frac{b / 2}{\alpha}\right)\right] .
\end{aligned}
$$

The condition for a double post bounce is that

$-\left(R_{\mathrm{P}}+R_{\mathrm{B}}\right)<y_{2}<\left(R_{\mathrm{P}}+R_{\mathrm{B}}\right)$.

Figure 7, obtained from Eq. (8), shows that this is satisfied for a range $\Delta x_{\mathrm{d}}$ of approximately $0.6 \mathrm{~mm}$, which according to Eq. (6) corresponds to a $\Delta \beta$ of $2.5^{\circ}$.

Using Eq. (3) we can calculate the corresponding $\theta$ for each $x$ and divide the inside quarter of the left goalpost into regions of different ball impact locations (see Fig. 8). The small margin for a double post bounce shows why one does not see it very frequently in soccer. The $\Delta \theta$ for the double post bounce for a penalty is indicated in green in Fig. 8 and can be calculated from $\Delta \beta=2.5^{\circ}$ and Eq. (3) to be only about $1.2^{\circ}$.

\section{- Ricochet off the right post}

The angle to the goal line, $\varepsilon$, relates to $\beta$ and $\varphi$ as (see Fig. 6)

$$
\varepsilon=2 \varphi-\beta .
$$

Further, the angles $\varphi, \beta$, and $\sigma$ relate as

$$
\varphi=\beta+\sigma .
$$

The angle $\sigma$ can be calculated from $y_{2}$ and the dimensions of the ball and goalpost as

$$
\sin \sigma=\frac{y_{2}}{R_{\mathrm{p}}+R_{\mathrm{B}}} .
$$

Inserting Eqs. (11) and (12) into Eq. (10), we get

$$
\varepsilon=2 \sigma+\beta=2 \sin ^{-1}\left(\frac{y_{2}}{R_{\mathrm{P}}+R_{\mathrm{B}}}\right)+\beta \text {. }
$$

Using Eq. (13), Fig. 9 shows the angle $\varepsilon$ for different values of $x$. For $\varepsilon>90^{\circ}$ the ball bounces off to the right side of 


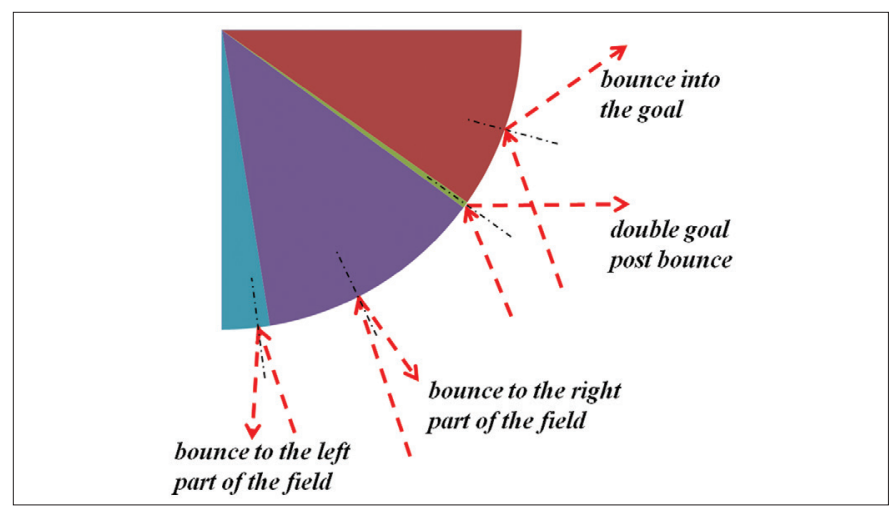

Fig. 8. Schematic of where the ball bounces after hitting the inside of the left goalpost. The ball ends up in the goal in the red region, double bounces in the green segment, and bounces back into the field for the purple and blue-green regions.

the field after hitting the right post. For $0^{\circ}<\varepsilon<90^{\circ}$ the ball moves back toward the left part of the field. The ball would move along the goal line, back to the left post for $\varepsilon=0^{\circ}$, and toward the net for negative values of $\varepsilon$. For $\varepsilon<-90^{\circ}$ the ball hits the very inside of the post and moves toward the right side net after the bounce off the right post.

\section{- Goal or no goal?}

To investigate how close the penalty was to making it into the goal for the win, we calculate the $y$-location $y_{1}{ }^{\prime}$ of the ball after it travels back the length of the goal toward the left post,

$$
y_{1}^{\prime}=b \tan \varepsilon+y_{2}
$$

Figure 10 shows $y_{1}{ }^{\prime}$ as a function of $x$ as calculated by inserting Eqs. (6) and (8) into Eq. (13) and then Eqs. (8) and (13) into Eq. (14). Note that the resulting expression is rather lengthy, but is easily calculated in a spreadsheet program.

The ball would hit the left post again, resulting in a triple bounce for

$-\left(R_{\mathrm{P}}+R_{\mathrm{B}}\right)<y_{1}^{\prime}<\left(R_{\mathrm{P}}+R_{\mathrm{B}}\right)$.

From the video one can conclude that it would have been very unlikely for the goalkeeper to get to the ball before it made it back to the left post. Hence, let us set the condition for a comparison penalty kick that would have won the game by just crossing the goal line without hitting the left post for a second time (see white dashed line in Fig. 2) as

$$
y_{1}^{\prime}<-\left(R_{\mathrm{P}}+R_{\mathrm{B}}\right)
$$

The corresponding $x$-value is denoted as $x_{\mathrm{C}}$ in Fig. 10.

Knowing $d=5.5 \mathrm{~m}$ and measuring $y_{\mathrm{p} 2} \approx 3 \mathrm{~m}$ from the video, we can calculate the position when the ball is at the level of the left post with Eq. (17) to approximately $y_{\mathrm{p} 1}=1.7 \mathrm{~m}$ (see Fig. 2)

$$
y_{\mathrm{p} 1}=\frac{b}{b+c} y_{\mathrm{p} 2} \text {. }
$$

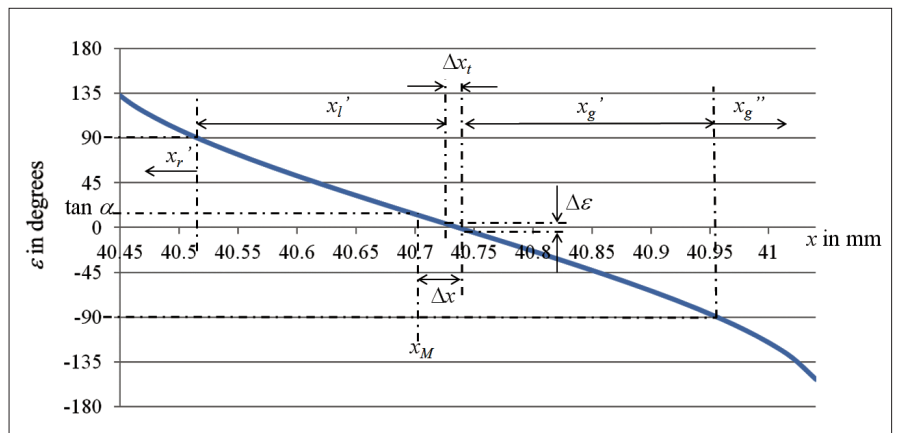

Fig. 9. Reflection angle $\varepsilon$ as a function of $x$ after the ball bounces off the left post. $x_{1}{ }^{\prime}$ and $x_{r}{ }^{\prime}$ are the $x$-values for which the ball bounces to the left $\left(x_{1}{ }^{\prime}\right)$ and right $\left(x_{r}{ }^{\prime}\right)$ part of the field after hitting the right post. For $x_{\mathrm{g}}{ }^{\prime}$ and $x_{\mathrm{g}}$ " the ball bounces into the goal without hitting the left post again. The ball moves toward the right side net for $x_{\mathrm{g}}$ ". The ball triple post bounces by hitting the left post again for $x$-values within $\Delta x_{t}$. The actual penalty in the game is denoted as $x_{M}$.

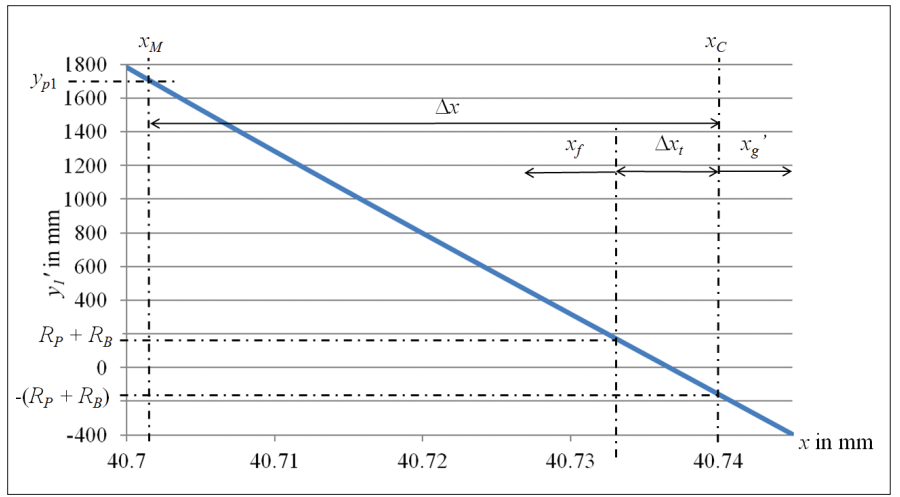

Fig. 10. $y_{1}$ ' as a function of $x$ as the ball is back at the left post. $x_{f}$ are the $x$-values for which the ball bounces back into the field after hitting the right post. The ball bounces into the goal without hitting the left post again for $x_{\mathrm{g}}$ ' and $x$-values. The ball triple bounces by hitting the left post again for $x$-values within $\Delta x_{t}$. The actual penalty and the hypothetical comparison penalty are denoted as $x_{M}$ and $x_{C}$ respectively. $\Delta x$ represents the difference in $x$ between the penalty and a successful double bounce penalty where the ball bounces off the left post, then the right post, and finally crosses the goal line just next to the left post.

The corresponding $x$-value, $x_{\mathrm{M}}$, can be obtained from Fig. 10 and compared to $x_{C}$ and the ball just slipping in the goal without hitting the left post again. The resulting difference, $\Delta x$, is slightly less than $40 \mu \mathrm{m}$. Hence, if the penalty would have been hit this distance further to the right of the left post, the ball would have gone into the net before the goalkeeper would have reached it. For reference, the average width of a human hair is about $80 \mu \mathrm{m}$ and thus we conclude:

\section{The penalty literally missed the goal by less than the width of a} hair!

The range $\Delta x_{\mathrm{t}}$ for a triple post bounce is even smaller and only approximately $7 \mu \mathrm{m}$. Hence, triple post bounce penalties are really rare. But, however unlikely, who is to say we will not see it at the most crucial time at some future World Cup Final? Sports events have a way of writing stories like these. 


\section{Conclusion}

The analysis presented here requires the use of a spreadsheet program, as well as the graphical representation of data, algebra, and trigonometry at a level accessible to high school and introductory college students. The results can be readily obtained from the figures; however, calculating the various $x$-values numerically from the boxed equations [Eq. (6), Eq. (8), Eq. (13), and Eq. (14)] cannot be done algebraically and would be a good exercise for a computational physics or mathematics course. Such a course could also calculate the $x$-values for the hypothetical case where a ball bounces back and forth, without being stopped by the goalkeeper or slowing down, hitting each goal post more than once.

As physics instructors, we try to encourage our students to apply what they learn in class in daily life. Sports science can provide many intriguing examples of physics in action. The double post penalty analysis presented here combines a few attractive features. The system was reasonably complex yet solvable using a simplified model. The result is surprising to many (most people we asked thought it missed by a much larger margin), and can provoke further discussions on why the system is so sensitive to small changes or how the simplifying assumptions impacted the result. Finally, the example invokes the passion of fans in that one does not often see a penalty shootout, let alone a potentially series-winning double post penalty in a high stakes game. The beauty of sports is that such rare events are more common than one may think. For example, one could imagine that an ice hockey enthusiast student could use a similar analysis to calculate the reflection of a puck from the goalpost. The attention and discussions spurred by "Deflategate"15-17 show the intrigue sport science can have for scientists, sports fans, the general public, and by extension students in our classroom. We hope that the analysis presented here is of interest to teachers and students with an interest in sport, and inspires them to hone their physics and mathematics skills leading to discussions with friends and family.

\section{Acknowledgments}

The author wants to acknowledge Michael Fitzgibbons and Elliot Mylott for their helpful feedback.

\section{References}

1. http://matchcenter.mlssoccer.com/matchcenter/201510-29-portland-timbers-vs-sporting-kansas-city/recap (accessed 07/29/2016).
2. http://matchcenter.mlssoccer.com/matchcenter/201510-29-portland-timbers-vs-sporting-kansas-city/boxscore (accessed 07/29/2016).

3. http://www.mlssoccer.com/post/2015/10/30/greatest-penaltykick-shootout-mls-history (the double post penalty is Sporting Kansas City's 9th penalty) (accessed 07/29/2016).

4. R. Cross, "Enhancing the bounce of a ball," Phys. Teach. 48, 450-452 (Oct. 2008).

5. R. Cross, "Grip-slip behavior of a bouncing ball," Am. J. Phys. 70, 1093-1102 (Nov. 2002).

6. R. Cross, "Bounce of a spinning ball near normal incidence," Am. J. Phys. 73, 914-920 (Oct. 2005).

7. A. Doménech, "A classical experiment revisited: The bounce of balls and superballs in three dimensions," Am. J. Phys. 73, 28-36 (Jan. 2005).

8. P. Knipp, "Bouncing balls that spin," Phys. Teach. 46, 95-96 (Feb. 2008).

9. A. M. Nathan, "The effect of spin on the flight of a baseball," Am. J. Phys. 76, 119-124 (Feb. 2008).

10. A. Štěpánek, “The aerodynamics of tennis balls-The topspin lob," Am. J. Phys. 56, 138-141 (Feb. 1988).

11. J. E. Goff and M. J. Carré, "Trajectory analysis of a soccer ball," Am. J. Phys. 77, 1020-1027 (Nov. 2009).

12. England-Germany, World Cup 2010, https://www.youtube. com/watch?v=HV4nc_sjW9Y (accessed 07/29/2016).

13. England-Germany, World Cup 1966, https://www.youtube. com/watch?v=0Uhe_11h3w8 (accessed 07/29/2016).

14. http://www.fifa.com/mm/document/affederation/ generic/81/42/36/lawsofthegame_2011_12_en.pdf (accessed 07/29/2016).

15. M. Lucibella, "Early fumbles in 'DeflateGate," APS News 24 (3), 1 (March 2015); https://www.aps.org/publications/ apsnews/201503/upload/March-2015.pdf .

16. Terrence Toepker, "Let's weigh in on 'Deflategate," Phys. Teach. 54, 338-339 (Sept. 2016).

17. Jack Blumenthal, Lauren Beljak, Dahlia-Marie Macatangay, Lilly Helmuth-Malone, Catharina McWilliams, and Sofia Raptis, "Deflategate': Time, temperature, and moisture effects on football pressure," Phys. Teach. 54, 340-343 (Sept. 2016).

Ralf Widenhorn received his Vordiplom in physics from the University of Konstanz, Germany, in 1997, and his PhD from Portland State University in the U.S. in 2005. He is currently an associate professor in the physics department at Portland State University. He has introduced various reforms to the introductory physics for the life sciences curriculum, and has published several journal articles describing biomedically inspired physics curriculum and lab activities. In 2013, he served as the local host at the annual summer meeting of the American Association of Physics Teachers. Besides physics educational research, Widenhorn has a background in semiconductor physics.

ralfw@pdx.edu 